\title{
The Homotopy Type of the Space of Degree 0 - Immersed Plane Curves
}

\author{
Hiroki KODAMA and Peter W. MICHOR \\ Erwin Schrödinger Institut \\ für Mathematische Physik \\ Boltzmanngasse 9 \\ A-1090 Wien - Austria \\ kodama@ms.u-tokyo.ac.jp \\ Fakultät für Mathematik \\ Universität Wien \\ Nordbergstrasse 15 \\ A-1090 Wien - Austria \\ Peter.Michor@univie.ac.at
}

Received: October 25, 2005

Accepted: December 22, 2005

\begin{abstract}
The space $B_{i}^{0}=\operatorname{Imm}^{0}\left(S^{1}, \mathbb{R}^{2}\right) / \operatorname{Diff}\left(S^{1}\right)$ of all immersions of rotation degree 0 in the plane modulo reparameterizations has homotopy groups $\pi_{1}\left(B_{i}^{0}\right)=\mathbb{Z}$, $\pi_{2}\left(B_{i}^{0}\right)=\mathbb{Z}$, and $\pi_{k}\left(B_{i}^{0}\right)=0$ for $k \geq 3$.
\end{abstract}

Key words: homotopy groups, immersed plane curves.

2000 Mathematics Subject Classification: Primary 58D10, 58D29, 55Q52.

\section{Introduction}

For an immersion $c: S^{1} \rightarrow \mathbb{R}^{2}$ the (rotation) degree is the winding number of $c^{\prime}: S^{1} \rightarrow \mathbb{R}^{2}$ around 0 . Let $\operatorname{Imm}^{k}\left(S^{1}, \mathbb{R}^{2}\right)$ denote the connected smooth Fréchet manifold of all immersions of degree $k$. It was shown in [4] that for $k \neq 0$ the space $\operatorname{Imm}^{k}\left(S^{1}, \mathbb{R}^{2}\right)$ contains a copy of $S^{1}$ as a smooth strong deformation retract and that the infinite dimensional orbifold $\operatorname{Imm}^{k}\left(S^{1}, \mathbb{R}^{2}\right) / \operatorname{Diff}^{+}\left(S^{1}\right)$ is contractible, where $\operatorname{Diff}^{+}\left(S^{1}\right)$ denotes the regular Fréchet Lie group of orientation preserving diffeomorphisms. The proof in [4] consists in expanding the classical proof of the theorem of Whitney and Graustein (see $[1,2,8]$ ) into the construction of an $S^{1}$-equivariant smooth deformation retraction. For $k=0$ this did not work.

PWM was supported by FWF Project P 17108. HK was supported by an IHÉS(EPDI)-JSPS fellowship. 
In this paper we treat the case $k=0$. In section 1 we first give simple argument which shows that $\pi_{1}\left(B^{0}\left(S^{1}, \mathbb{R}^{2}\right)\right)$ contains $\mathbb{Z}$. In section 2 we give a more involved proof that $\operatorname{Imm}^{0}\left(S^{1}, \mathbb{R}^{2}\right)$ is homotopy equivalent to $S^{1}$. In section 3 we show that factoring out $\operatorname{Diff}^{+}\left(S^{1}\right)$ gives a fibration with homotopically trivially embedded fiber, and then the homotopy sequence shows that $\pi_{1}\left(B^{0,+}\left(S^{1}, \mathbb{R}^{2}\right)\right)=\mathbb{Z}, \pi_{2}\left(B^{0,+}\left(S^{1}, \mathbb{R}^{2}\right)\right)=\mathbb{Z}$, and $\pi_{k}\left(B^{0,+}\left(S^{1}, \mathbb{R}^{2}\right)\right)=0$ for $k>2$. Factoring out the larger group $\operatorname{Diff}\left(S^{1}\right)$ gives a two-sheeted covering and the final result.

\section{A simple proof that $\mathbb{Z} \subseteq \pi_{1}\left(B^{0}\left(S^{1}, \mathbb{R}^{2}\right)\right)$}

Proposition 1.1. $\operatorname{Imm}^{0}\left(S^{1}, \mathbb{R}^{2}\right) / \operatorname{Diff}^{+}\left(S^{1}\right)$ is not contractible.

Proof. We shall view a curve $c \in \operatorname{Imm}\left(S^{1}, \mathbb{R}^{2}\right)$ as a $2 \pi$-periodic plane valued function. A smooth function $a=a(c, \quad): \mathbb{R} \rightarrow \mathbb{R}$ is called an argument of a curve $c$ if

$$
\frac{c^{\prime}(\theta)}{\left|c^{\prime}(\theta)\right|}=\exp (i a(\theta))
$$

it is unique up to addition of an integer multiple of $2 \pi$. If the curve $c$ has degree $k$ then $a(\theta+2 \pi)-a(\theta)=2 k \pi$. Thus, a curve $c$ is in $\operatorname{Imm}^{0}\left(S^{1}, \mathbb{R}^{2}\right)$ if and only if some (any) argument of $c$ is $2 \pi$-periodic. For a curve $c \in \operatorname{Imm}^{0}\left(S^{1}, \mathbb{R}^{2}\right)$, we define the average argument $\alpha(c) \in S^{1}$ by

$$
\alpha(c)=\exp \left(\frac{i}{l(c)} \int_{0}^{2 \pi} a(c, \theta)\left|c^{\prime}(\theta)\right| d \theta\right)
$$

which does not depend on the choice of $a(c, \quad)$ and defines a well-defined smooth mapping $\alpha: \operatorname{Imm}^{0}\left(S^{1}, \mathbb{R}^{2}\right) \rightarrow S^{1}$. Also, since any argument $a$ of a degree 0 curve is $2 \pi$-periodic, $\alpha(c)$ is invariant under the action of $\operatorname{Diff}^{+}\left(S^{1}\right)$. So we can view $\alpha$ as a map

$$
\alpha: B^{0,+}\left(S^{1}, \mathbb{R}^{2}\right)=\operatorname{Imm}^{0}\left(S^{1}, \mathbb{R}^{2}\right) / \operatorname{Diff}^{+}\left(S^{1}\right) \rightarrow S^{1} .
$$

For $\varphi \in S^{1} \subset \mathbb{C}=\mathbb{R}^{2}$, the rotation map $\varphi: \mathbb{R}^{2} \rightarrow \mathbb{R}^{2}$ act on $B^{0,+}\left(S^{1}, \mathbb{R}^{2}\right)$ and obviously

$$
\alpha(\varphi \cdot c)=\varphi \cdot \alpha(c) .
$$

So choosing a free orbit $S^{1} . C$ for the rotation action of $S^{1}$ on $B^{0,+}\left(S^{1}, \mathbb{R}^{2}\right)$, the composition

$$
S^{1} \cdot C \hookrightarrow \operatorname{Imm}^{0}\left(S^{1}, \mathbb{R}^{2}\right) / \operatorname{Diff}^{+}\left(S^{1}\right) \stackrel{\alpha}{\longrightarrow} S^{1}
$$

equals the identity on $S^{1}$, thus $\pi_{1}\left(S^{1}\right)=\mathbb{Z} \subset \pi_{1}\left(B^{0,+}\left(S^{1}, \mathbb{R}^{2}\right)\right)$. 
Moreover, $\alpha(c(-\quad))=-\alpha(c)$ implies that $\alpha$ factors as follows, where the vertical arrows are 2-sheeted coverings:

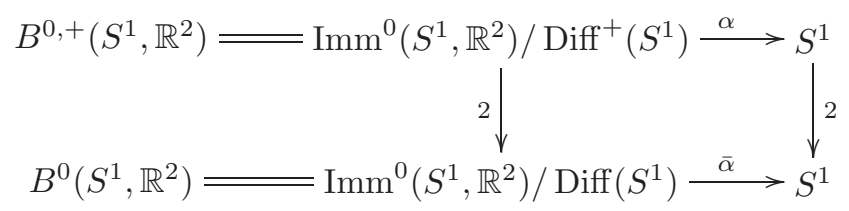

Thus we also get in a similar way $\pi_{0}\left(S^{1}\right)=\mathbb{Z} \subset \pi_{0}\left(B^{0}\left(S^{1}, \mathbb{R}^{2}\right)\right)$.

\section{The homotopy type of $\operatorname{Imm}^{0}\left(S^{1}, \mathbb{R}^{2}\right)$}

Proposition 2.1. The space $\operatorname{Imm}^{0}\left(S^{1}, \mathbb{R}^{2}\right)$ of degree 0 immersions in the plane is homotopy equivalent to $S^{1}$.

Proof. This will follow from 2.2-2.5 below.

2.2. Let $\operatorname{Imm}^{0, *}\left(S^{1}, \mathbb{R}^{2}\right):=\left\{c \in \operatorname{Imm}^{0}\left(S^{1}, \mathbb{R}^{2}\right): c(0)=0\right\}$. Clearly we have $\operatorname{Imm}^{0}\left(S^{1}, \mathbb{R}^{2}\right) \cong \operatorname{Imm}^{0, *}\left(S^{1}, \mathbb{R}^{2}\right) \times \mathbb{R}^{2}$ and $\operatorname{Imm}^{0}\left(S^{1}, \mathbb{R}^{2}\right) \sim \operatorname{Imm}^{0, *}\left(S^{1}, \mathbb{R}^{2}\right)$, where $\cong$ denotes homeomorphic and $\sim$ homotopy equivalent. Let us define a map

$$
\begin{gathered}
\Phi: \operatorname{Imm}^{0, *} \rightarrow C^{\infty}\left(S^{1}, \mathbb{R}_{+}\right) \times C^{\infty}\left(S^{1}, S^{1}\right) \\
\Phi(c)(\theta)=\left(\left|c_{\theta}(\theta)\right|, \frac{c_{\theta}(\theta)}{\left|c_{\theta}(\theta)\right|}\right)=:(v(\theta), e(\theta)) .
\end{gathered}
$$

The map $\Phi$ is injective. For $(v, e)=\Phi(c)$, the winding number of $e$ equals the degree 0 of $c$ and thus $\int_{0}^{2 \pi} v \cdot e d \theta=0$.

Lemma. The length of the image of $e$ is greater than $\pi$.

Proof. If not, there exists a number $r \in \mathbb{R}$ such that

$$
\exp (i r) \in \operatorname{Im}(e) \subset \exp (i[r-\pi / 2, r+\pi / 2]) .
$$

Then, $\langle\exp (i r), e(\theta)\rangle$ is nonnegative for any $\theta$ and strictly positive for some $\theta$. Therefore $\int_{0}^{2 \pi}\langle\exp (i r), v \cdot e\rangle d \theta>0$. This contradicts

$$
\int_{0}^{2 \pi}\langle\exp (i r), v \cdot e\rangle d \theta=\left\langle\exp (i r), \int_{0}^{2 \pi} v \cdot e d \theta\right\rangle=\langle\exp (i r), 0\rangle=0 .
$$

2.3. Let us define the set

$$
C_{>\pi}^{\infty, 0}\left(S^{1}, S^{1}\right)=\left\{e \in C^{\infty}\left(S^{1}, S^{1}\right): \operatorname{deg}(e)=0, \operatorname{length}(\operatorname{Im}(e))>\pi\right\}
$$

and consider the map

$$
\operatorname{pr}_{2} \circ \Phi: \operatorname{Imm}^{0, *}\left(S^{1}, S^{1}\right) \rightarrow C_{>\pi}^{\infty, 0}\left(S^{1}, S^{1}\right),
$$

where $\mathrm{pr}_{2}$ denotes the second projection. 
Lemma. The map $\operatorname{pr}_{2} \circ \Phi: \operatorname{Imm}^{0, *}\left(S^{1}, S^{1}\right) \rightarrow C_{>\pi}^{\infty, 0}\left(S^{1}, S^{1}\right)$, is surjective, has contractible fibers, admits a global smooth section, and is a homotopy equivalence.

Proof. For a map $e \in C_{>\pi}^{\infty, 0}\left(S^{1}, S^{1}\right)$, there exist points $\theta_{1}, \theta_{2}, \theta_{3}$ such that $0 \in \operatorname{int}\left(\left[e\left(\theta_{1}\right), e\left(\theta_{2}\right), e\left(\theta_{3}\right)\right]\right)$, where $[\cdot, \cdot, \cdot \cdot]$ denotes the convex hull of three points. Let $v_{1} \in C^{\infty}\left(S^{1}, \mathbb{R}_{>0}\right)$ be a map such that $\int_{0}^{2 \pi} v_{1} d \theta=1$ and $v_{1}(\theta)$ is close to 0 if $\theta$ is not close to $\theta_{1}$. Then $\int_{0}^{2 \pi} v_{1} \cdot e d \theta$ is close to $e\left(\theta_{1}\right)$. We also define $v_{2}$ and $v_{3}$ similarly, so that

$$
0 \in \operatorname{int}\left(\left[\int_{0}^{2 \pi} v_{1} \cdot e d \theta, \int_{0}^{2 \pi} v_{2} \cdot e d \theta, \int_{0}^{2 \pi} v_{3} \cdot e d \theta\right]\right) .
$$

Therefore there exist positive numbers $a_{1}, a_{2}, a_{3}$ with

$$
a_{1} \int_{0}^{2 \pi} v_{1} \cdot e d \theta+a_{2} \int_{0}^{2 \pi} v_{2} \cdot e d \theta+a_{3} \int_{0}^{2 \pi} v_{3} \cdot e d \theta=0 .
$$

Define $c$ by

$$
c(\theta)=\int_{0}^{\theta}\left(a_{1} v_{1}(u)+a_{2} v_{2}(u)+a_{3} v_{3}(u)\right) e(u) d u .
$$

Then $c$ is in $\operatorname{Imm}^{0, *}$ and $\left(\mathrm{pr}_{2} \circ \Phi\right)(c)=e$, which means that $\mathrm{pr}_{2} \circ \Phi$ is surjective.

We next show that for any $e \in C_{>\pi}^{\infty, 0}\left(S^{1}, S^{1}\right)$, the inverse image $\left(\operatorname{pr}_{2} \circ \Phi\right)^{-1}(e)$ is contractible. Namely, let $V(e) \subset C^{\infty}\left(S^{1}, \mathbb{R}_{+}\right)$be given by

$$
V(e)=\left\{v \in C^{\infty}\left(S^{1}, \mathbb{R}_{+}\right): \int_{0}^{2 \pi} v \cdot e d \theta=0\right\},
$$

an open convex subset of the linear subspace $\left\{v \in C^{\infty}\left(S^{1}, \mathbb{R}\right): \int_{0}^{2 \pi} v \cdot e d \theta=0\right\} \subset$ $C^{\infty}\left(S^{1}, \mathbb{R}\right)$. Thus $V(e)$ is contractible for each $e$. Moreover, $V(e)$ is homeomorphic to $\left(\operatorname{pr}_{2} \circ \Phi\right)^{-1}(e)$ by the map $\operatorname{pr}_{1} \circ \Phi:\left(p_{2} \circ \Phi\right)^{-1}(e) \rightarrow V(e)$.

For fixed $\theta_{1}, \theta_{2}, \theta_{3}$ the construction above works for each $e \in C_{>\pi}^{\infty, 0}\left(S^{1}, S^{1}\right)$ for which 0 is contained in the interior of the convex hull of $e\left(\theta_{1}\right), e\left(\theta_{2}\right), e\left(\theta_{3}\right)$; these $e$ form an open set in $C_{>\pi}^{\infty, 0}\left(S^{1}, S^{1}\right)$ on which we get a continuous (even smooth) section of $p_{2} \circ \Phi$. Open sets like that cover $C_{>\pi}^{\infty, 0}\left(S^{1}, S^{1}\right)$. So we get smooth local sections whose domains cover the base. Since the base is open in a nuclear Fréchet space, it is smoothly paracompact (see $[3,16.10]$ ) we can use convexity of all fibers and a smooth partition of unity on the base $C_{>\pi}^{\infty, 0}\left(S^{1}, S^{1}\right)$ to construct a global smooth section $s$.

Finally, since all fibers are convex, there is a smooth strong fiber preserving deformation retraction of $\operatorname{Imm}^{0, *}\left(S^{1}, S^{1}\right)$ onto the image the global section $s$.

2.4. To study the topology of $C_{>\pi}^{\infty, 0}\left(S^{1}, S^{1}\right)$, we introduce the set of $2 \pi$-periodic functions

$$
C^{\infty, p}(\mathbb{R}, \mathbb{R})=\left\{c \in C^{\infty}(\mathbb{R}, \mathbb{R}): c(\theta+2 \pi)=c(\theta)\right\} .
$$


For $c \in C^{\infty, p}(\mathbb{R}, \mathbb{R})$ let $\operatorname{Var}(c)=\max c-\min c$ and let $\operatorname{Ave}(c)=\frac{1}{2 \pi} \int_{0}^{2 \pi} c d \theta$. For $k \geq 0, C_{>k}^{\infty, p}(\mathbb{R}, \mathbb{R})=\left\{c \in C^{\infty, p}(\mathbb{R}, \mathbb{R}): \operatorname{Var}(c)>k\right\}$. Define a diffeomorphism $g: C_{>0}^{\infty, p}(\mathbb{R}, \mathbb{R}) \rightarrow C_{>\pi}^{\infty, p}(\mathbb{R}, \mathbb{R})$ by

$$
g(c)=\frac{\operatorname{Var}(c)+\pi}{\operatorname{Var}(c)}(c-\operatorname{Ave}(c))+\operatorname{Ave}(c) .
$$

The diffeomorphism $g$ satisfies $g(c(+2 n \pi))=g(c)(+2 n \pi)$, thus induces the diffeomorphism

$$
\tilde{g}: C_{>0}^{\infty, 0}\left(S^{1}, S^{1}\right) \rightarrow C_{>\pi}^{\infty, 0}\left(S^{1}, S^{1}\right),
$$

where $C_{>0}^{\infty, 0}\left(S^{1}, S^{1}\right)$ denotes the set of nonconstant smooth maps of degree 0 in $C^{\infty}\left(S^{1}, S^{1}\right)$.

2.5. We consider now the evaluation ev 1 at $1 \in S^{1}$ whose fiber at $1 \in S^{1}$ is the smooth manifold of based smooth loops of degree 0 in $S^{1}$, with the constant loop 1 deleted:

$$
C^{\infty, 0}\left(\left(S^{1}, 1\right),\left(S^{1}, 1\right)\right) \backslash\{1\} \hookrightarrow C_{>0}^{\infty, 0}\left(S^{1}, S^{1}\right) \stackrel{\mathrm{ev}_{1}}{\longrightarrow} S^{1}
$$

Lemma. The map ev $\mathrm{ev}_{1}: C_{>0}^{\infty, 0}\left(S^{1}, S^{1}\right) \rightarrow S^{1}$ is a smooth trivial fibration with a global section and smoothly contractible fibers. Moreover, it is a homotopy equivalence.

Proof. A smooth section $s: S^{1} \rightarrow C_{>0}^{\infty, 0}\left(S^{1}, S^{1}\right)$ of $\mathrm{ev}_{1}$ is given by $s(\varphi)(\theta)=\varphi$. $\exp (i \operatorname{Im}(\theta))$. The fiber of $\operatorname{ev}_{1}$ over $\varphi$ is the space $C^{\infty, 0}\left(\left(S^{1}, 1\right),\left(S^{1}, \varphi\right)\right) \backslash\{\varphi\}$ consisting of all non-constant smooth loops of degree 0 mapping 1 to $\varphi$, which is diffeomorphic to the fiber $C^{\infty, 0}\left(\left(S^{1}, 1\right),\left(S^{1}, 1\right)\right) \backslash\{1\}$ via multiplication by $\varphi$.

It remains to show that the fiber $C^{\infty, 0}\left(\left(S^{1}, 1\right),\left(S^{1}, 1\right)\right) \backslash\{1\}$ is contractible. Via lifting to the universal cover, $C^{\infty, 0}\left(\left(S^{1}, 1\right),\left(S^{1}, 1\right)\right)$ is diffeomorphic to the space $\left\{f \in C^{\infty, p}(\mathbb{R}, \mathbb{R}): f(0)=0\right\}$ of periodic functions mapping 0 to 0 . Via Fourier expansion $f(t)=\sum_{n \in \mathbb{Z}} a_{n} \exp ($ int $)$ this is isomorphic to the space of all rapidly decreasing complex sequences $\left(a_{k}\right)_{k \in \mathbb{Z}}$ with $\overline{a_{k}}=a_{-k}$ and $\sum_{k} a_{k}=0$. This space is isomorphic to the space $\mathfrak{s}$ of rapidly decreasing sequences $\left(b_{n}\right)_{n \geq 1}$ by $a_{n}=b_{n}$ for $n \geq 1, a_{-n}=\bar{b}_{n}$, and $a_{0}=2 \operatorname{Re}\left(\sum_{n \geq 1} b_{n}\right)$.

Now we have to show that this is still contractible if we remove the constant sequence 0 . Then it is homotopy equivalent to its intersection with the sphere in $\ell^{2}$, i.e., to the space $S:=\left\{b \in \mathfrak{s}: \sum_{n \geq 1} b_{n}^{2}=1\right\}$. But this is contractible by a standard argument which is explained on page 513 of [3] for the space of finite sequences. Namely, consider the homotopy $A: \mathfrak{s} \times[0,1] \rightarrow \mathfrak{s}$ through isometries which is given by $A_{0}=\mathrm{Id}$ and by

$$
\begin{aligned}
A_{t}\left(b_{1}, b_{2}, b_{3}, \ldots\right)=\left(b_{1}, \ldots, b_{n-2}, b_{n-1} \cos \theta_{n}(t), b_{n-1} \sin \theta_{n}(t),\right. \\
\left.\quad b_{n} \cos \theta_{n}(t), b_{n} \sin \theta_{n}(t), b_{n+1} \cos \theta_{n}(t), b_{n+1} \sin \theta_{n}(t), \ldots\right)
\end{aligned}
$$

for $\frac{1}{n+1} \leq t \leq \frac{1}{n}$, where $\theta_{n}(t)=\varphi(n((n+1) t-1)) \frac{\pi}{2}$ for a fixed smooth function $\varphi: \mathbb{R} \rightarrow \mathbb{R}$ which is 0 on $(-\infty, 0]$, grows monotonely to 1 in $[0,1]$, and equals 1 on 
$[1, \infty)$. The mapping $A$ is Lipschitz continuous for each seminorm $\|b\|_{k}=\sup \left\{\left|b_{n}\right| n^{k}\right.$ : $n \geq 1\}$ of $\mathfrak{s}$ with constant $2^{k}$, and is isometric for $\ell^{2}$. Then $A_{1 / 2}\left(b_{1}, b_{2}, \ldots\right)=$ $\left(b_{1}, 0, b_{2}, 0, \ldots\right)$ is in $\mathfrak{s}_{\text {odd }}$, and on the other hand $A_{1}\left(b_{1}, b_{2}, \ldots\right)=\left(0, b_{1}, 0, b_{2}, 0, \ldots\right)$ is in $\mathfrak{s}_{\text {even. }}$. This is a variant of a homotopy constructed by [6]. Now $A_{t} \mid S$ for $0 \leq t \leq 1 / 2$ is a homotopy on $S$ between the identity and $A_{1 / 2}(S) \subset \mathfrak{s}_{\text {odd }}$. The latter set is contractible, for example in a stereographic chart.

2.6. If we put together all mappings constructed above we get the following commutative diagram where we indicate isomorphism $\cong$, homotopy equivalence $\sim$, or 2 -sheeted covering 2 , and a free orbit $S^{1} \cdot c$ for the rotation action on $\mathrm{Imm}^{0}$ :

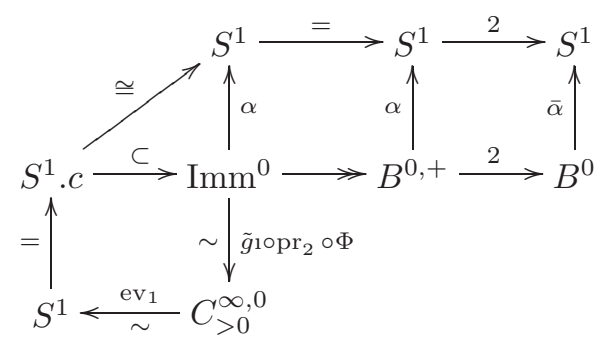

\section{The homotopy type of $B^{0}\left(S^{1}, \mathbb{R}^{2}\right)$}

Proposition 3.1. The mapping $\operatorname{Imm}^{0}\left(S^{1}, \mathbb{R}^{2}\right) \rightarrow B^{0,+}\left(S^{1}, \mathbb{R}^{2}\right)$ is a (Serre) fibration.

Proof. First we replace $\operatorname{Imm}^{0}\left(S^{1}, \mathbb{R}^{2}\right)$ by the subset $\operatorname{Imm}_{a}^{0}\left(S^{1}, \mathbb{R}^{2}\right)$ consisting of all immersions which are parametrized by scaled arc-length which is a strong deformation retract, see $[4,2.6]$. The normalizer of the $\operatorname{Diff}^{+}\left(S^{1}\right)$-action on it is just the action of $S^{1}$ which shifts the initial point. We have to show that for any compactly generated space $P$ and a homotopy $h:[0,1] \times P \rightarrow B^{0,+}$ whose initial value $h(0, \quad)$ admits a continuous lift there exists a continuous lift of the whole homotopy:

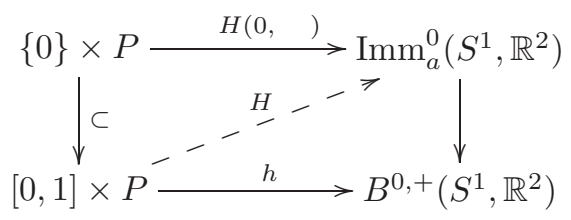

To get the lift $H$ we just have to specify the initial point coherently from $H(0, p)(1)$ over $[0,1] \ni t \mapsto h(t, p)$.

For that we need a description of the elements in $B^{0,+}\left(S^{1}, \mathbb{R}^{2}\right)$. A point $C$ in it can be described by the following data:

For some $n$ and $i=1, \ldots, n$, there are open sets $U_{i}=U_{i}(C) \subseteq \mathbb{R}^{2}$, smooth functions $f_{i}=f_{i}(C): U_{i} \rightarrow \mathbb{R}$ such that $f_{i}^{-1}(0)=: C_{i}$ is a component $C_{i}$ of $C$ with 
$\operatorname{grad}\left(f_{i}\right)$ is a unit vector field with flow lines unit speed straight lines passing orthogonally through $C_{i}$ in such a way that for $x \in C_{i}$ the frame consisting of $\operatorname{grad}\left(f_{i}\right)(x)$ and the unit tangent to $C_{i}$ at $x$ is positively oriented. The unparameterized smooth oriented 1-manifolds $C_{1}, C_{2}, \ldots, C_{n}$ (in that order) describe $C$. Note that there is a choice for the $U_{i}$ and their cyclic order, but then the $f_{i}$ are unique.

For every $p \in P$ the initial point $H(0, p)(1)$ lies in some component $h(0, p)_{i}$ of $h(0, p)$, and we may move it orthogonally along $\operatorname{grad}\left(f_{i}(h(t, p))\right)$ to get a coherent choice of initial points. This takes care of the lift $H$.

Lemma 3.2. The fiber $\operatorname{Diff}^{+}\left(S^{1}\right)$ maps homotopically trivial into the fibration

$$
\operatorname{Imm}^{0}\left(S^{1}, \mathbb{R}^{2}\right) \rightarrow B^{0,+}\left(S^{1}, \mathbb{R}^{2}\right) .
$$

Proof. As in the proof of 3.1 we consider the space $\operatorname{Imm}_{a}^{0}\left(S^{1}, \mathbb{R}^{2}\right)$ of degree 0 immersions with constant speed parameterizations. Let $c$ be the unit speed parameterized horizontal figure eight, and consider the diagram where $c^{*}(f)=c \circ f$ :

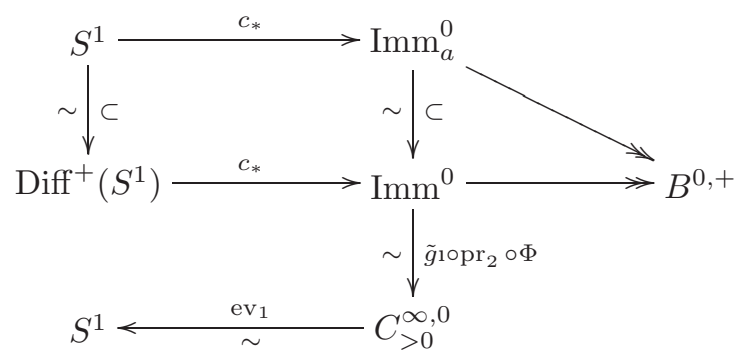

We have to show that the mapping from the upper left $S^{1}$ to the lower left $S^{1}$ is nullhomotopic. It is essentially (suppressing $\tilde{g}^{-1}$ ) given by $\beta \mapsto \frac{c^{\prime}(\beta)}{\left|c^{\prime}(\beta)\right|}$. From the figure
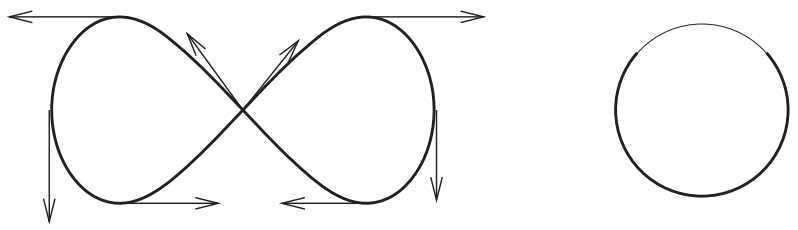

we see that this mapping covers everything below the northern polar region twice and avoids the northern polar region, so it is nullhomotopic.

Corollary 3.3. We have the following homotopy groups:

$$
\begin{array}{ll}
\pi_{1}\left(B^{0,+}\left(S^{1}, \mathbb{R}^{2}\right)\right)=\mathbb{Z}, & \pi_{1}\left(B^{0}\left(S^{1}, \mathbb{R}^{2}\right)\right)=\mathbb{Z}, \\
\pi_{2}\left(B^{0,+}\left(S^{1}, \mathbb{R}^{2}\right)\right)=\mathbb{Z}, & \pi_{2}\left(B^{0}\left(S^{1}, \mathbb{R}^{2}\right)\right)=\mathbb{Z}, \\
\pi_{k}\left(B^{0,+}\left(S^{1}, \mathbb{R}^{2}\right)\right)=0, & \pi_{k}\left(B^{0}\left(S^{1}, \mathbb{R}^{2}\right)\right)=0 \quad \text { for } k>2 .
\end{array}
$$


Proof. By 3.1 we have the long exact homotopy sequence

$$
\cdots \rightarrow \pi_{k}\left(S^{1}\right) \stackrel{0}{\longrightarrow} \pi_{k}\left(\operatorname{Imm}_{a}^{0}\right) \rightarrow \pi_{k}\left(B^{0,+}\right) \rightarrow \pi_{k-1}\left(S^{1}\right) \rightarrow \cdots
$$

and by section 2 the space $\operatorname{Imm}_{a}^{0}$ is homotopy equivalent to $S^{1}$. This gives the homotopy groups of $\left.B^{0,+}\left(S^{1}, \mathbb{R}^{2}\right)\right)$. Since $\left.B^{0,+}\left(S^{1}, \mathbb{R}^{2}\right)\right) \rightarrow B^{0}\left(S^{1}, \mathbb{R}^{2}\right)$ ) is a twosheeted covering, we can also read of the homotopy groups of $\left.B^{0,+}\left(S^{1}, \mathbb{R}^{2}\right)\right)$.

Acknowledgements. PM thanks the audience of the lecture course SS 2005 and Peter Zvengrowksi for helpful discussions. HK thanks the Erwin Schrödinger Institute for the warm hospitality during his stay in Vienna.

\section{References}

[1] W. C. Graustein, A new form of the four-vertex theorem, Mh. Math. Physics 43 (1936), 381-384.

[2] _ Extensions of the four-vertex theorem, Trans. Amer. Math. Soc. 41 (1937), no. 1, 9-23.

[3] A. Kriegl and P. W. Michor, The convenient setting of global analysis, Mathematical Surveys and Monographs, vol. 53, American Mathematical Society, Providence, RI, 1997.

[4] P. W. Michor and D. Mumford, Riemannian geometries on spaces of plane curves, J. Eur. Math. Soc. 8 (2006), 1-48.

[5] - Vanishing geodesic distance on spaces of submanifolds and diffeomorphisms, Doc. Math. 10 (2005), 217-245 (electronic), available at arXiv:math.DG/0409303.

[6] T. R. Ramadas, On the space of maps inducing isomorphic connections, Ann. Inst. Fourier (Grenoble) 32 (1982), no. 1, viii, 263-276.

[7] G. W. Whitehead, Elements of homotopy theory, Graduate Texts in Mathematics, vol. 61, Springer-Verlag, New York, 1978.

[8] H. Whitney, Regular families of curves. I, Proc. Nat. Acad. Sci USA 18 (1932), 275-278; II, Proc. Nat. Acad. Sci USA 18 (1932), 340-342. 